Original Research Paper

\title{
Constructing Gibbs Measure in a Rigorous Way
}

\author{
${ }^{1}$ Farida Kachapova and ${ }^{2}$ Ilias Kachapov \\ ${ }^{1}$ Department of Mathematical Sciences, Auckland University of Technology, Auckland, New Zealand \\ ${ }^{2}$ Examination Academic Services, University of Auckland, Auckland, New Zealand
}

\author{
Article history \\ Received: 31-08-2019 \\ Revised: $29-09-2019$ \\ Accepted: 28-10-2019 \\ Corresponding Author: \\ Farida Kachapova \\ Department of Mathematical \\ Sciences, Auckland University \\ of Technology, \\ New Zealand \\ Email: farida.kachapova@aut.ac.nz
}

\begin{abstract}
Equilibrium statistical mechanics studies mathematical models for physical systems with many particles interacting with an external force and with one another. In this paper we describe an interaction model that generalizes several of these models in one model. An infinite model is constructed as the limiting case of finite interaction models, that is as a thermodynamic limit. The key point in constructing a thermodynamic limit is a proof of existence of the limiting probability measure (Gibbs measure). Traditional proofs use DLR formalism and are quite complicated. Here we explain a more transparent and more constructive proof for the case of high temperatures. The paper provides a detailed, step-by-step rigorous construction of a statistical model and corresponding proofs. The paper also includes a version of the central limit theorem for a random field transformed by a renormalization group, in a special case of the interaction model.
\end{abstract}

Keywords: Infinite Particle System, Gibbs Modification, Radius of Interaction, Thermodynamic Limit, Semi-Invariant

\section{Introduction}

Mathematical models of statistical mechanics are described in many publications; see, for example, Dobrushin (1968), Kashapov (1977), Malyshev (1980), Malyshev and Minlos (1991), Baxter (2008), and Yang et al. (2017). Well-known models include Ising model, Potts model, Heisenberg model, $X Y$ model and $n$-vector model. In Kachapova and Kachapov (2016) we introduced a so called interaction model; we further developed this concept in Kachapova and Kachapov (2017). The interaction model describes a physical system at high temperature with many particles interacting with an external force and with one another. In this paper we improve the mathematical concept of the interaction model by giving a more general and detailed definition and we show that all the aforementioned models are particular cases of the interaction model.

A general model of a physical system with many particles is based on a probability theory. When an infinite model is constructed as a limit of finite models (a thermodynamic limit), there occurs a problem of existence of its probability measure (Gibbs measure). The general mathematical proof of existence of Gibbs measure was constructed using Dobrushin-LanfordRuelle (DLR) approach. It was described in several books: Ruelle (1999), Preston (1976), Malyshev and
Minlos (1991), Friedli and Velenik (2017) and in most general form in Georgii (2011). The general existence proof is quite complicated and involves topology, cluster expansion and conditional probabilities.

In this paper we explain a straightforward existence proof for the case of high temperatures, which involves only basic probability, calculus and combinatorics and is more transparent and more constructive than the general proof.

Section 1 introduces a probability space and characteristics for a finite interaction model, and gives a definition of this model. In Section 2 we introduce some notations and state Existence Theorem (about existence of Gibbs measure). Next in this section we define an infinite interaction model using the Gibbs measure; then we briefly justify that the Ising model, Potts model and $n$-vector model are particular cases of the interaction model (and so are Heisenberg model and $X Y$-model, since they are particular cases of the $n$-vector model).

In Section 3 we state a convergence theorem for a random field transformed by a renormalization group. This theorem is stated for a special case of the interaction model constructed in this paper but the proof from our earlier paper Kachapova and Kachapov (2016) still holds.

The remaining Sections 4, 5 and 6 give a detailed proof of the Existence Theorem. In particular, Section 4 considers some estimations in graphs. In Section 5 graph-related estimations are applied to semi-invariants and their series. In Section 6 the probability $P_{N}(A)$ of an 
event $A$ in a finite model is written as a series of semiinvariants. In Section 6 the proof of the Existence Theorem is finalised by proving absolute and uniform convergence of the aforementioned series for $P_{N}(A)$ and taking a limit as $N \rightarrow \infty$.

\section{Finite Interaction Model}

\subsection{Gibbs Modification}

Gibbs modification is used to modify a given probability in order to describe interaction between particles.

For any probability measure $P$ on $(\Omega, \Sigma)$ denote $\langle\cdot\rangle_{P}$ the expectation with respect to $P$.

\section{Definition 1.1}

Suppose $P$ is a probability measure on $(\Omega, \Sigma)$ and $U$ is a bounded random variable on $(\Omega, \Sigma)$.

Gibbs modification of the probability measure $P$ by the random variable $U$ is denoted $P_{U}$ and is defined as follows. For any event $A \in \Sigma$ :

$$
P_{U}(A)=\frac{\left\langle I_{A} e^{U}\right\rangle_{P}}{\left\langle e^{U}\right\rangle_{P}},
$$

where $I_{A}$ denotes the indicator of the event $A$.

\section{Lemma 1.2}

In conditions of the previous definition:

1) $P_{U}$ is a probability measure on $(\Omega, \Sigma)$;

2) for any random variable $Y$ on $(\Omega, \Sigma)$,

$$
\langle Y\rangle_{P_{U}}=\frac{\left\langle Y e^{U}\right\rangle_{P}}{\left\langle e^{U}\right\rangle_{P}} .
$$

Proof is well-known and can be found, for example, in Kachapova and Kachapov (2017).

\subsection{Construction of Probability Space}

\section{Definition 1.3}

We introduce some objects that will be used to construct an interaction model.

1) Fix natural numbers $v \geq 1$ and $\eta \geq 1$. Consider a $v$ dimensional integer lattice:

$$
\mathbb{Z}^{v}=\left\{\boldsymbol{t}=\left(t_{1}, \ldots, t_{v}\right) \mid t_{i} \in \mathbb{Z}, i=1, \ldots, v\right\}
$$

with the distance between any two points $\boldsymbol{s}, \boldsymbol{t} \in \mathbb{Z}^{v}$ defined by:

$$
\|\boldsymbol{s}-\boldsymbol{t}\|=\sum_{i=1}^{v}\left|s_{i}-t_{i}\right|
$$

2) For any $\boldsymbol{t} \in \mathbb{Z}^{v}$ we denote $\Omega_{t}=\mathbb{R}^{\eta}$ and $\Sigma_{t}$ the Borel $\sigma$ algebra on $\mathbb{R}^{\eta}$; we fix a probability measure $P_{t}$ on $\left(\Omega_{t}, \Sigma_{t}\right)$.

We call $\left\{P_{t} \mid t \in \mathbb{Z}^{v}\right\}$ the initial probabilities.

\section{Definition 1.4}

1) By the Hahn-Kolmogorov theorem, for any integer $k>1$ and distinct $\boldsymbol{t}_{1}, \ldots, \boldsymbol{t}_{k} \in \mathbb{Z}^{v}$ there exists a probability space $\left(\Omega_{t_{1}} \times \ldots \times \Omega_{t_{k}}, \Sigma_{t_{1} \ldots t_{k}}, P_{t_{1} \ldots t_{k}}\right)$ such that $\Sigma_{t_{1} \ldots t_{k}}$ is the $\sigma$-algebra generated by elements of the Cartesian product $\Sigma_{\boldsymbol{t}_{1}} \times \ldots \times \Sigma_{\boldsymbol{t}_{k}}$ and $P_{\boldsymbol{t}_{1} \ldots \boldsymbol{t}_{k}}$ is a unique probability measure with the property:

$$
P_{t_{1} \ldots t_{k}}\left(F_{1} \times \ldots \times F_{k}\right)=P_{t_{1}}\left(F_{1}\right) \cdot \ldots \cdot P_{t_{k}}\left(F_{k}\right)
$$

for any $F_{i} \in \Sigma_{\boldsymbol{t}_{i}}(i=1, \ldots, k)$.

2) By the Kolmogorov extension theorem, there exists a probability space $\left(\Omega, \Sigma, P_{0}\right)$, where the elements are described as follows.

a) $\Omega=\left(\mathbb{R}^{\eta}\right)^{\mathbb{Z}^{v}}$, that is $\Omega=\left\{\omega \mid \omega: \mathbb{Z}^{v} \rightarrow \mathbb{R}^{\eta}\right\}$. An element $\omega$ of $\Omega$ is called a configuration and is interpreted as a state of a physical system.

b) $\Sigma$ is the $\sigma$-algebra generated by sets of the form $\{\omega \in \Omega \mid \omega(\boldsymbol{t}) \in F\}$ for all $\boldsymbol{t} \in \mathbb{Z}^{v}$ and $F \in \Sigma_{\boldsymbol{t}}$.

c) $P_{0}$ is a unique probability measure on $(\Omega, \Sigma)$ such that for any integer $k>1$, distinct $\boldsymbol{t}_{1}, \ldots, \boldsymbol{t}_{k} \in \mathbb{Z}^{v}$ and $F_{1} \in \Sigma_{\boldsymbol{t}_{1}}, \ldots, F_{k} \in \Sigma_{\boldsymbol{t}_{k}}$ :

$$
P_{0}\left(\omega\left(\boldsymbol{t}_{1}\right) \in F_{1}, \ldots, \omega\left(\boldsymbol{t}_{k}\right) \in F_{k}\right)=P_{t_{1} \ldots t_{k}}\left(F_{1} \times \ldots \times F_{k}\right) .
$$

Thus, $P_{0}$ is uniquely defined by the initial probabilities $\left\{P_{t} \mid \boldsymbol{t} \in \mathbb{Z}^{v}\right\}$.

3) For any $\boldsymbol{t} \in \mathbb{Z}^{v}$ a function $X_{t}: \Omega \rightarrow \mathbb{R}^{\eta}$ is defined by the following:

$$
X_{t}(\omega)=\omega(t)
$$

$X$ denotes $\left\{X_{t} \mid \boldsymbol{t} \in \mathbb{Z}^{v}\right\}$.

For the rest of the paper we fix the objects $\mathbb{Z}^{v}, \mathbb{R}^{\eta}$, $\left(\Omega, \Sigma, P_{0}\right)$ and $X$ from these definitions. For brevity we denote $\langle\cdot\rangle_{P_{0}}$ as $\langle\cdot\rangle_{0}$. 


\section{Lemma 1.5}

$\left\{X_{t} \mid t \in \mathbb{Z}^{v}\right\}$ is an independent random vector field on the probability space $\left(\Omega, \Sigma, P_{0}\right)$.

\section{Proof}

Proof follows from the definitions.

\section{Definition 1.6}

1) Consider a graph $(V, E)$, where the set of vertices $V$ is a finite subset of $\mathbb{Z}^{v}$ and $E$ is the set of edges; each edge can be regarded as a pair of distinct vertices (there are no loops). The length of each edge is the distance between its end vertices.

The graph $(V, E)$ is called 1-connected if it is connected and the length of each of its edges equals 1 .

2) For a finite set $B \subset \mathbb{Z}^{v}$ define its size $S(B)$ as the minimum number of edges of 1-connected graphs $(V, E)$ such that $B \subseteq V$.

\section{Remark}

For any finite set $B$ there is a cube in $\mathbb{Z}^{v}$ containing $B$; this cube with all its edges of length 1 is a 1 connected graph. So $S(B)$ is always defined.

\section{Notation 1.7}

For any non-empty finite subset $B$ of $\mathbb{Z}^{v}$ denote $\Sigma_{B}$ the $\sigma$-algebra generated by sets of the form $\{\omega \in \Omega \mid \omega(\boldsymbol{t}) \in F\}$ for all $\boldsymbol{t} \in B$ and $F \in \Sigma_{\boldsymbol{t}}$.

\section{Definition 1.8}

Here we introduce three characteristics $\beta, r, \Phi$ of an interaction model and a set $\mathfrak{B}$.

1) $\beta \in \mathbb{R}^{\eta}, \beta \geq 0$. This is thermodynamic $\beta$ inversely proportional to the temperature of the physical system.

2) $r \in \mathbb{R}, r \geq 1$. $\mathrm{r}$ is called the radius of interaction.

3) Denote $\mathfrak{B}=\left\{B \subset \mathbb{Z}^{v} \mid 1 \leq S(B) \leq r\right\}$.

4) For each $B \in \mathfrak{B}, \Phi_{B}$ is a random variable on $\left(\Omega, \Sigma_{B}\right)$ that satisfies the condition:

$$
\left|\Phi_{B}\right| \leq \beta
$$

$\Phi$ denotes $\left\{\Phi_{B} \mid B \in \mathfrak{B}\right\} . \Phi$ is called the potential of the system.

\section{Remarks}

1) Clearly, if $B \in \mathfrak{B}$, then $B$ is finite.

2) Two particles (represented by points of $\mathbb{Z}^{v}$ ) interact only if they both belong to some set $B(B \in \mathfrak{B})$, and $\Phi_{B}$ is the interaction energy of all elements of $B$.
3) In our definition of $\mathfrak{B}$ we have only sets with $S(B) \geq 1$, i.e. sets with at least two elements. The general case (that includes one-element sets $B$ ) can be reduced to this case by a single Gibbs modification as shown in Kachapova and Kachapov (2017), pg. 343-344.

\subsection{Finite Interaction Model}

\section{Definition 1.9}

A finite interaction model with characteristics $\beta, r$, $\Phi$ and $\left\{P_{t} \mid \boldsymbol{t} \in \mathbb{Z}^{v}\right\}$ (as in Definition 1.3.2) is a sequence $\left(\Lambda, U_{\Lambda}, P_{\Lambda}\right)$ of three objects defined as follows.

1) $\Lambda$ is an arbitrary finite subset of the lattice $\mathbb{Z}^{v}$.

2) A function $U_{\Lambda}: \Omega \rightarrow \mathbb{R}$ is called the interaction energy of $\Lambda$ and is defined by the following:

$$
\text { for any } \omega \in \Omega, U_{\Lambda}(\omega)=\sum_{B \in \mathfrak{B}, B \subseteq \Lambda} \Phi_{B}(\omega),
$$

where the set $\mathfrak{B}$ is defined in Definition 1.8.

$U_{\Lambda}(\omega)$ characterizes the energy of configuration $\omega$ in $\Lambda$.

3) Denote $P_{\Lambda}=\left(P_{0}\right)_{U_{\Lambda}}$, the Gibbs modification of the probability $P_{0}$ (from Definition 1.4 ) by the interaction energy $U_{\Lambda}$.

In Kachapova and Kachapov (2017) it was shown that $\left(\Omega, \Sigma, P_{\Lambda}\right)$ is a probability space.

\section{Infinite Interaction Model}

\subsection{Existence Theorem}

\section{Definition 2.1}

1) For any $B \in \mathfrak{B}$ denote $l(B)$ the number of sets $C \in \mathfrak{B}$ that intersect with $B$.

2) Denote $L=\max \{l(B) \mid B \in \mathfrak{B}\}$.

So $L=\max \left\{l(B) \mid B \subset \mathbb{Z}^{v}\right.$ and $\left.1 \leq S(B) \leq r\right\}$.

Clearly, $L$ depends only on $v$ and $r$.

\section{Theorem 2.2}

In the finite interaction model the number $L$ is defined and

$$
L \leq(4 v)^{2 r-1}(r+1)
$$

This theorem will be proven in Section 4 .

\section{Remarks}

1) $L$ is a characteristic of interaction in the physical system. 
2) We do not aim to obtain the most accurate estimate of $L$. However, it is not hard to get more accurate estimates in particular cases. For example, when $r=1$ like in the Ising, Potts and $n$ vector models, $L=4 v-1$ because in these models only neighboring points in $\mathbb{Z}^{v}$ interact, i.e. each set in $\mathfrak{B}$ is a set of two neighboring points.

\section{Definition 2.3}

Define $\beta_{0}=\frac{1}{50 L(8 v)^{2 r}}$, where $L$ was introduced in Definition 2.1.2.

Clearly, $\beta_{0}$ depends only on $v$ and $r$.

Notation 2.4.

For any $N \in \mathbb{N}$ define a cube $\Lambda_{N}$ in $\mathbb{Z}^{v}$ :

$\Lambda_{N}=\left\{\boldsymbol{t}=\left(t_{1}, \ldots, t_{v}\right) \in \mathbb{Z}^{v}|| t_{i} \mid \leq N\right.$ for each $\left.i=1, \ldots, v\right\}$.

\section{Notation 2.5}

Suppose $Q$ is a non-empty finite subset of $\mathbb{Z}^{v}$.

1) Denote $\mathbb{N}_{Q}=\left\{N \in \mathbb{N} \mid Q \subseteq \Lambda_{N}\right\}$. For any $N \in \mathbb{N}_{Q}$ :

- $\left(\Lambda_{N}, U_{\Lambda_{N}}, P_{\Lambda_{N}}\right)$ is the finite interaction model with characteristics $\beta, r, \Phi$ and $\left\{P_{t} \mid \boldsymbol{t} \in \mathbb{Z}^{v}\right\}$ from Definition 1.9;

- $\quad$ for brevity denote $U_{N}=U_{\Lambda_{N}}$ and $P_{N}=P_{\Lambda_{N}}$.

2) For any $A \in \Sigma_{Q}$ define:

$$
P_{\beta, Q}(A)=\lim _{N \rightarrow \infty} P_{N}(A) .
$$

\section{Theorem 2.6 (Existence Theorem)}

Suppose $0 \leq \beta \leq \beta_{0}$ and $Q$ is a non-empty finite subset of $\mathbb{Z}^{v}$. Then the following hold.

1) For any $A \in \Sigma_{Q}$ the limit in (1) exists.

2) $P_{\beta, Q}$ is a probability measure on $\left(\Omega, \Sigma_{Q}\right)$.

This theorem will be proven in Sections 5 and 6 .

\section{Corollary 2.7}

Suppose $0 \leq \beta \leq \beta_{0}$. There exists a unique probability measure $P_{\beta}$ on $(\Omega, \Sigma)$ such that for any non-empty finite set $Q \subset \mathbb{Z}^{v}$ and any $A \in \Sigma_{Q}$ :

$$
P_{\beta}(A)=P_{\beta, Q}(A) .
$$

Proof

For any integer $k>1$, distinct $\boldsymbol{t}_{1}, \ldots, \boldsymbol{t}_{k} \in \mathbb{Z}^{v}$ and $D \in \Sigma_{t_{1} \ldots t_{k}}$ define:

$$
P_{\beta, t_{1} \ldots t_{k}}(D)=P_{\beta,\left\{t_{1}, \ldots t_{k}\right\}}\left(\left\{\omega \in \Omega \mid\left(\omega\left(\boldsymbol{t}_{1}\right), \ldots, \omega\left(\boldsymbol{t}_{k}\right)\right) \in D\right\}\right) .
$$

By Theorem 2.6.2), each $P_{\beta,\left\{t_{1}, \ldots, t_{k}\right\}}$ is a probability measure on $\left(\Omega, \Sigma_{\left\{t_{1}, \ldots, t_{k}\right\}}\right)$. Therefore the formula (2) defines a probability measure on $\left(\Omega_{t_{1}} \times \ldots \times \Omega_{t_{k}}, \Sigma_{t_{1} \ldots t_{k}}\right)$ as a projection probability, for any distinct $\boldsymbol{t}_{1}, \ldots, \boldsymbol{t}_{k} \in \mathbb{Z}^{v}$.

In particular, for any $F_{i} \in \Sigma_{\boldsymbol{t}_{i}}(i=1, \ldots, k)$ :

$$
P_{\beta, \boldsymbol{t}_{1} \ldots t_{k}}\left(F_{1} \times \ldots \times F_{k}\right)=P\left(\bigcap_{i=1}^{K}\left\{\omega \in \Omega \mid \omega\left(\boldsymbol{t}_{i}\right) \in F_{i}\right\}\right) .
$$

These probabilities satisfy the following two consistency conditions.

1) For any distinct $\boldsymbol{t}_{1}, \ldots, \boldsymbol{t}_{k} \in \mathbb{Z}^{v}$, permutation $\pi$ of $\{1,2, \ldots, k\}$ and $F_{i} \in \Sigma_{\boldsymbol{t}_{i}}(i=1, \ldots, k)$ :

$$
P_{\beta, t_{\pi(1)} \ldots t_{\pi(k)}}\left(F_{\pi(1)} \times \ldots \times F_{\pi(k)}\right)=P_{\beta, t_{1} \ldots t_{k}}\left(F_{1} \times \ldots \times F_{k}\right) .
$$

2) For any distinct $\boldsymbol{t}_{1}, \ldots, \boldsymbol{t}_{k}, \boldsymbol{t}_{k+1} \in \mathbb{Z}^{v}$ and $F_{i} \in \Sigma_{\boldsymbol{t}_{i}}(i=1, \ldots, k)$

$$
P_{\beta, t_{1} \ldots t_{k}}\left(F_{1} \times \ldots \times F_{k}\right)=P_{\beta, t_{1} \ldots t_{k} t_{k+1}}\left(F_{1} \times \ldots \times F_{k} \times \mathbb{R}^{\eta}\right) .
$$

Hence the corollary follows from the Kolmogorov extension theorem.

The probability measure $P_{\beta}$ is called the Gibbs measure.

\subsection{Infinite Interaction Model}

\section{Definition 2.8}

Suppose $0 \leq \beta \leq \beta_{0}$. An infinite interaction model with characteristics $\beta, r, \Phi$ and $\left\{P_{\boldsymbol{t}} \mid \boldsymbol{t} \in \mathbb{Z}^{v}\right\}$ is the probability space $\left(\Omega, \Sigma, P_{\beta}\right)$, where $P_{\beta}$ is the probability measure from Corollary 2.7 .

The probability space $\left(\Omega, \Sigma, P_{\beta}\right)$ is also called the thermodynamic limit or macroscopic limit of the finite interaction models $\left(\Lambda_{N}, U_{N}, P_{N}\right)$ as $N \rightarrow \infty$.

Next we show that several well-known models can be considered as particular cases of the interaction model.

\subsection{Potts Model as a Particular Case of Interaction Model}

The standard Potts model is based on the interaction energy (also called Hamiltonian):

$$
U_{\Lambda}=\sum_{\substack{s, t \in \Lambda \\|k-t|=1}} J_{s t} \delta\left(X_{s}, X_{t}\right),
$$


where $J_{\text {st }}$ are coupling constants and the random variables $X_{t}$ (interpreted as site colors) take values 1 , $2, \ldots, q$ with equal probabilities;

$$
\delta(x, y)=\left\{\begin{array}{l}
1 \text { if } x=y \\
0 \text { if } x \neq y
\end{array}\right.
$$

Applying Definition 1.8, we take $\eta=1$, an arbitrary $v \geq 1$, an arbitrary $\beta \geq 0, r=1$ and the probabilities $\left\{P_{\boldsymbol{t}} \mid \boldsymbol{t} \in \mathbb{Z}^{v}\right\}$ such that for any Borel set $A$ :

$$
P_{t}(A)=\frac{|A \cap\{1,2, \ldots q\}|}{q},
$$

where the numerator is the cardinality of the set $A \cap\{1,2, \ldots, q\}$.

Since $r=1$, then $\mathfrak{B}=\left\{\{\boldsymbol{s}, \boldsymbol{t}\} \subset \mathbb{Z}^{v} \mid\|\boldsymbol{s}-\boldsymbol{t}\|=1\right\}$ and for any $B \in \mathfrak{B}$ with $B=\{\boldsymbol{s}, \boldsymbol{t}\}$ we define:

$$
\Phi_{B}(\omega)=\beta K_{s t} \delta(\omega(\boldsymbol{s}), \omega(\boldsymbol{t})),
$$

where $\left|K_{s t}\right| \leq 1$.

When $0 \leq \beta \leq \beta_{0}$ and $N \rightarrow \infty$ (i.e. $\Lambda \rightarrow \mathbb{Z}^{v}$ ), the Potts model becomes a particular case of the infinite interaction model.

\subsection{Ising Model as a Particular Case of Interaction Model}

The Ising model is equivalent to the Potts model for $q=$ 2. The Ising model is based on the interaction energy:

$$
U_{\Lambda}=\sum_{\substack{s, t \in \Lambda \\\|s-\|=1}} J_{s t} X_{s} X_{t}-\sum_{t \in \Lambda} h_{t} X_{t}
$$

where $J_{s t}, h_{t}$ are constants and random variables $X_{t}$ take values \pm 1 with equal probabilities.

To eliminate the second sum in $U_{\Lambda}$ (representing an external magnetic field) we apply a single Gibbs modification described in Kachapova and Kachapov (2017), pg.343-344 and get the probabilities $\left\{P_{\boldsymbol{t}} \mid \boldsymbol{t} \in \mathbb{Z}^{v}\right\}$ such that for any Borel set $A$ :

$$
P_{t}(A)=\frac{1}{e^{h_{t}}+e^{-h_{t}}} \sum_{\substack{x \in A \\ x= \pm 1}} e^{-x h_{t}} .
$$

Applying Definition 1.8 we take $\eta=1$, an arbitrary $v \geq 1$, an arbitrary $\beta \geq 0$, the probabilities $\left\{P_{\boldsymbol{t}} \mid \boldsymbol{t} \in \mathbb{Z}^{v}\right\}$ given by (3), and $r=1$.

Then $\mathfrak{B}=\left\{\{\boldsymbol{s}, \boldsymbol{t}\} \subset \mathbb{Z}^{v} \mid\|\boldsymbol{s}-\boldsymbol{t}\|=1\right\}$ and for any $B \in \mathfrak{B}$ with $B=\{\boldsymbol{s}, \boldsymbol{t}\}$ we define:

$$
\Phi_{B}(\omega)=\beta K_{s t} \omega(s) \omega(t)
$$

where $\left|K_{s t}\right| \leq 1$.

When $0 \leq \beta \leq \beta_{0}$ and $N \rightarrow \infty$ (i.e. $\Lambda \rightarrow \mathbb{Z}^{v}$ ), the Ising model becomes a particular case of the infinite interaction model.

\section{5. $\mathrm{N}$-Vector Model}

In the $n$-vector model classical spins $\boldsymbol{s}_{i}\left(\boldsymbol{i} \in \mathbb{Z}^{v}\right)$ are $n$ dimensional vectors with unit length; the interaction energy of the $n$-vector model is given by:

$$
U_{\Lambda}=-J \sum_{\substack{i, j \in \Lambda \\\|i-j\|=1}} s_{i} \cdot s_{j},
$$

where $J$ is a constant and $\cdot$ denotes scalar product.

Applying Definition 1.8 we take $\eta=n$, an arbitrary $v \geq 1$, an arbitrary $\beta \geq 0$, and $r=1$.

For each $\boldsymbol{t} \in \mathbb{Z}^{v}$, the probability measure $P_{t}$ is uniformly distributed on the unit sphere:

$$
S^{\eta-1}=\left\{\boldsymbol{x}=\left(x_{1}, \ldots, x_{\eta}\right) \in \mathbb{R}^{\eta} \mid x_{1}^{2}+\ldots+x_{\eta}^{2}=1\right\},
$$

that is for any set $A \in \Sigma_{t}$ :

$$
P_{t}(A)=\frac{\operatorname{Area}\left(A \cap S^{\eta-1}\right)}{\operatorname{Area}\left(S^{\eta-1}\right)} .
$$

Since $r=1$, then $\mathfrak{B}=\left\{\{\boldsymbol{s}, \boldsymbol{t}\} \subset \mathbb{Z}^{v} \mid\|\boldsymbol{s}-\boldsymbol{t}\|=1\right\}$ and for any $B \in \mathfrak{B}$ with $B=\{\boldsymbol{s}, \boldsymbol{t}\}$ we define:

$$
\Phi_{B}(\omega)=-J \omega(s) \cdot \omega(\boldsymbol{t})
$$

When $0 \leq \beta \leq \beta_{0}$ and $N \rightarrow \infty$ (i.e. $\Lambda \rightarrow \mathbb{Z}^{v}$ ), the $n$ vector model becomes a particular case of the infinite interaction model.

Particular cases of the $n$-vector model are the Ising model $(n=1), X Y$ model $(n=2)$, and Heisenberg model $(n=3)$.

\section{Convergence Theorem for a Field Transformed by Renorm-Group}

The concept of renormalization group was introduced by Kadanoff (1966).

\section{Definition 3.1}

Fix a natural number $k>1$ and a real number $\alpha \geq v$.

For each $\boldsymbol{s}=\left(s_{1}, s_{2}, \ldots, s_{v}\right) \in \mathbb{Z}^{v}$ consider a cube $C_{s}^{k}$ of width $k$ with vertex $k s$ : 


$$
C_{s}^{k}=\left\{\boldsymbol{t} \in \mathbb{Z}^{v} \mid k s_{i} \leq t_{i}<k\left(s_{i}+1\right), i=1,2, \ldots v\right\}
$$

A renormalization group, or renorm-group $(R G)$ with parameters $k$ and $\alpha$ is a transformation that assigns to each random field $\left\{Z_{t} \mid t \in \mathbb{Z}^{v}\right\}$ a new random field $\left\{Y_{s}^{(k)} \mid \boldsymbol{s} \in \mathbb{Z}^{v}\right\}$ given by:

$$
Y_{s}^{(k)}=k^{-\frac{\alpha}{2}} \sum_{\boldsymbol{t} \in C_{s}^{k}}\left(Z_{t}-\left\langle Z_{\boldsymbol{t}}\right\rangle_{\beta}\right)
$$

The renormalization group is a scaling transformation (e.g., from atomic to molecular level).

We are interested in the limiting distribution of the result $Y_{s}^{(k)}$ of the $R G$ transformation of the random field $\left\{X_{t} \mid \boldsymbol{t} \in \mathbb{Z}^{v}\right\}$ as $k \rightarrow \infty$ (as the distance scale infinitely increases). We find the limiting distribution for a special case of the interaction model.

\section{Definition 3.2}

Denote $W$ the set of all vectors from the standard basis in $\mathbb{R}^{v}$.

A special interaction model is the infinite interaction model with the following five constraints.

1) $\eta=1$

2) $r=1$. Then each set $B \in \mathfrak{B}$ consists of two points at distance 1, that is $B$ has the form $B=\{\boldsymbol{s}, \boldsymbol{s}+\boldsymbol{u}\}$, $\boldsymbol{u} \in W$.

3) The initial probabilities $P_{t}$ are the same for all $\boldsymbol{t} \in \mathbb{Z}^{v}$.

4) Transition invariance:

there is a set of Borel functions $\varphi_{\boldsymbol{u}}(\boldsymbol{u} \in W)$ such that for each $B \in \mathfrak{B}$,

$$
\begin{aligned}
& \Phi_{B}(\omega)=\varphi_{u}(\omega(\boldsymbol{s}), \omega(\boldsymbol{s}+\boldsymbol{u})), \\
& \text { where } B=\{\boldsymbol{s}, \boldsymbol{s}+\boldsymbol{u}\} \text { and } \boldsymbol{u} \in W
\end{aligned}
$$

5) Each $X_{t}$ satisfies Carleman condition:

$$
\sum_{k=1}^{\infty}\left(\left\langle X_{t}^{2 k}\right\rangle_{0}\right)^{-\frac{1}{2 k}}=\infty .
$$

This condition means: the probabilities of large values for the random variable are close to 0. Any bounded random variable satisfies the Carleman condition; so do variables with normal and exponential distributions.

Both Ising and Potts models are particular cases of the special interaction model.

\section{Theorem 3.3 (Convergence Theorem)}

There is a constant $C>0$ depending only on $v$ and initial probabilities $\left\{P_{\boldsymbol{t}} \mid \boldsymbol{t} \in \mathbb{Z}^{v}\right\}$ such that the following holds for any special interaction model with $\beta \leq \mathrm{C}$.

Suppose a renorm-group with parameters $k$ and $\alpha$ transforms the random field $\left\{X_{\boldsymbol{t}} \mid \boldsymbol{t} \in \mathbb{Z}^{v}\right\}$ into a random field $\left\{Y_{s}^{(k)} \mid \boldsymbol{s} \in \mathbb{Z}^{v}\right\}$.

1) If $\alpha>v$, then the field $Y_{s}^{(k)} \rightarrow 0$ in mean square as $k \rightarrow \infty$.

2) If $\alpha=v$, then as $k \rightarrow \infty$, the random field $\left\{Y_{s}^{(k)} \mid \boldsymbol{s} \in \mathbb{Z}^{v}\right\}$ converges in distribution to an independent random field with Gaussian distribution.

Each of the variables of the limiting field has 0 expectation and the positive variance given by:

$$
V=\sum_{n=0}^{\infty} \beta^{n} V_{n},
$$

where each coefficient $V_{n}$ is a finite sum of semiinvariants of $X_{\boldsymbol{t}}$ and $\Phi_{B}$ with respect to $P_{0} ; \boldsymbol{t} \in \mathbb{Z}^{v}, B \in \mathfrak{B}$.

Proof of the Convergence Theorem is given on our paper Kachapova and Kachapov (2016).

Clearly, the limiting distribution is not always Gaussian. We believe that in Theorem 3.3 the Carleman condition (stated in the definition of a special interaction model) is essential for the limiting distribution to be Gaussian.

The Convergence Theorem is a generalization of the Central Limit Theorem (CLT); while the limit in CLT is a single variable, the limit in our theorem is a random field and we found its joint distribution.

\section{Graph-Associated Estimations}

This section is devoted to proof of Theorem 2.2.

\subsection{Tracks in Graphs}

\section{Definition 4.1}

A track is a sequence $t r=\left(\boldsymbol{t}_{0}, \boldsymbol{t}_{1}, \ldots, \boldsymbol{t}_{n}\right)$ of points in $\mathbb{Z}^{v}$ such that $\boldsymbol{t}_{0}=\boldsymbol{t}_{n}$ and $\left\|\boldsymbol{t}_{i}-\boldsymbol{t}_{i-1}\right\|=1, i=1, \ldots, n$.

\section{Lemma 4.2}

Fix $\boldsymbol{t}_{0} \in \mathbb{Z}^{v}$ and $n>1$. The number of tracks of the form $\left(\boldsymbol{t}_{0}, \boldsymbol{t}_{1}, \ldots, \boldsymbol{t}_{n}\right)$ is not greater than $(2 v)^{n-1}$.

\section{Proof}

The first element $\boldsymbol{t}_{0}$ is fixed. There are $2 v$ choices for element $\boldsymbol{t}_{1}$, at most $2 v$ choices for element $\boldsymbol{t}_{2}$, at most $2 v$ 
choices for element $t_{3}, \ldots$, and only one choice for element $\boldsymbol{t}_{n}$, since $\boldsymbol{t}_{n}=\boldsymbol{t}_{0}$. So the total number of tracks is at most $(2 v)^{n-1}$.

\section{Definition 4.3}

Suppose $B$ is a finite subset of $\mathbb{Z}^{v}$ and $m=S(B)$. There is a 1-connected graph $(V, E)$ such that $B \subseteq V$, and the number of edges in this graph is minimal and equals $m$. We denote $G_{B}$ the first (in lexicographic order) of such graphs and call it the associated graph of $B$.

\section{Lemma 4.4}

Suppose $B$ is a finite subset of $\mathbb{Z}^{v}$ and $m=S(B)$. Then the graph $G_{B}$ is a tree with $m$ edges and $m+1$ vertices.

\section{Proof}

A tree is a connected graph with no cycles. The definition of size implies that $G_{B}$ is connected.

If $G_{B}$ has a cycle, then we can remove one edge of the cycle thus reducing the number of edges while the graph remains connected with the same set of vertices. That contradicts the choice of $G_{B}$ as the graph with minimal number of edges.

By the definition $G_{B}$ has $m$ edges. Since it is a tree, the number of its vertices is $m+1$.

\section{Definition 4.5}

Suppose $B$ is a finite subset of $\mathbb{Z}^{v}$ and $m=S(B)$. We create the associated track $\operatorname{tr}_{B}$ of $B$ in the following two steps:

1. We obtain a graph $G_{B}^{\prime}$ from the associated graph $G_{B}$ by adding for every its edge another edge with the same ends.

2. The new graph $G_{B}^{\prime}$ has $2 m$ edges and each of its vertices has an even degree. Therefore there is an Eulerian path in $G_{B}^{\prime}$, i.e. a closed path that includes every edge of the graph exactly once. We denote $\left(e_{1}, e_{2}, \ldots, e_{2 m}\right)$ the first (in lexicographic order) of such paths.

We define $t r_{B}$ as the corresponding sequence $\left(\boldsymbol{t}_{0}, \boldsymbol{t}_{1}\right.$, $\left.\boldsymbol{t}_{2}, \ldots, \boldsymbol{t}_{2 m}\right)$ of vertices, that is $\left\{\boldsymbol{t}_{i-1}, \boldsymbol{t}_{i}\right\}$ are the ends of $e_{i}$ $(i=1,2, \ldots, 2 m)$.

\section{Definition 4.6}

Suppose $B \in \mathfrak{B}$. Its associated track has the form $t r_{B}=\left(\boldsymbol{t}_{0}, \boldsymbol{t}_{1}, \boldsymbol{t}_{2}, \ldots, \boldsymbol{t}_{2 m}\right)$, where $m=S(B)$ and $m \leq r$.

We define the extended track $t r_{B}^{\prime}=\left(\boldsymbol{t}_{0}, \boldsymbol{t}_{1}, \boldsymbol{t}_{2}, \ldots, \boldsymbol{t}_{2 m}, \boldsymbol{t}_{2 m+1}, \ldots, \boldsymbol{t}_{2 r}\right)$ of $B$ by the following:

- for $i=1, \ldots, r-m$ each point $\boldsymbol{t}_{2 m+i}$ is obtained from $\boldsymbol{t}_{2 m+i-1}$ by increasing its first coordinate by 1 ;

- for $i=r-m+1, \ldots, 2 r-2 m$ each point $\boldsymbol{t}_{2 m+i}$ is obtained from $\boldsymbol{t}_{2 m+i-1}$ by decreasing its first coordinate by 1 .

\section{Lemma 4.7}

1) For any finite subset $B$ of $\mathbb{Z}^{v}, t r_{B}$ is a track.

2) For any $B \in \mathfrak{B}, t r_{B}^{\prime}$ is a track.

\section{Proof}

Follows from the definitions.

\subsection{Estimations in Graphs}

\section{Lemma 4.8}

Suppose $t r=\left(\boldsymbol{t}_{0}, \boldsymbol{t}_{1}, \boldsymbol{t}_{2}, \ldots, \boldsymbol{t}_{2 r}\right)$ is a track. Then there are at most $2^{2 r-1}$ sets $C \in \mathfrak{B}$ such that $\boldsymbol{t}_{0} \in C$ and $t r_{C}^{\prime}=t r$.

\section{Proof}

Since the track $t r$ is a closed path, we have $\boldsymbol{t}_{0}=\boldsymbol{t}_{2 r}$. Denote $V_{1}=\left\{\boldsymbol{t}_{1}, \boldsymbol{t}_{2}, \ldots, \boldsymbol{t}_{2 r-1}\right\}$. If a set $C \in \mathfrak{B}$ has $t r_{C}^{\prime}=t r$, then:

$$
C \backslash\left\{\boldsymbol{t}_{0}\right\} \subseteq V_{1}
$$

Since $V_{1}$ has at most $2^{2 r-1}$ different subsets, then there are at most $2^{2 r-1}$ sets $C$ with property (4).

\section{Lemma 4.9}

Suppose $t_{0} \in \mathbb{Z}^{v}$. Then there are at most $(4 v)^{2 r-1}$ sets $C \in \mathfrak{B}$ with $\boldsymbol{t}_{0} \in C$.

\section{Proof}

For each set $C \in \mathfrak{B}$ with $\boldsymbol{t}_{0} \in C$ we have an extended track $t r_{C}^{\prime}$; since a track is a closed path, we can assume $t r_{C}^{\prime}$ starts at $\boldsymbol{t}_{0}$. So there is a correspondence between sets $C \in \mathfrak{B}$ with $\boldsymbol{t}_{0} \in C$ and tracks of the form $\left(\boldsymbol{t}_{0}, \boldsymbol{t}_{1}, \ldots, \boldsymbol{t}_{2 r}\right)$. By Lemma 4.8 at most $2^{2 r-1}$ sets correspond to each track and by Lemma 4.2 the total number of such tracks starting at $\boldsymbol{t}_{0}$ is not greater than $(2 v)^{2 r-1}$. Therefore the number of sets $C \in \mathfrak{B}$ with $\boldsymbol{t}_{0} \in C$ is not greater than $2^{2 r-1}(2 v)^{2 r-1}=(4 v)^{2 r-1}$.

\subsection{Proof of Theorem 2.2}

\section{Proof}

Fix an arbitrary $B \in \mathfrak{B}$. It is sufficient to show:

$$
l(B) \leq(4 v)^{2 r-1}(r+1) .
$$

Since $S(B) \leq r$, we have $|B| \leq r+1$. Any set $C \in \mathfrak{B}$ that intersects with $B$ contains one of the $(r+1)$ elements of $B$. By Lemma 4.9, for each element $\boldsymbol{t}_{0} \in B$ there are at most $(4 v)^{2 r-1}$ sets $C \in \mathfrak{B}$ with $\boldsymbol{t}_{0} \in C$. So the total number of sets $C \in \mathfrak{B}$ that intersect with $B$ is not greater than $(4 v)^{2 r-1}(r+1)$ 


\section{Technical Definitions and Estimations}

The rest of the paper is devoted to proof of the Existence Theorem (Theorem 2.6). For the rest of the paper we fix $\Omega, \Sigma$, characteristics $\beta, r, \Phi,\left\{P_{t} \mid \boldsymbol{t} \in \mathbb{Z}^{v}\right\}$, a finite set $Q \subset \mathbb{Z}^{v}$ with size $q=S(Q)$ and an event $A \in \Sigma_{Q}$. We will consider only integers $N \in \mathbb{N}_{Q}$.

Section 5 contains some technical concepts and lemmas, which lead to the proof of the Existence Theorem in Section 6.

\subsection{Semi-Invariants}

Denote $\langle\cdot, \ldots,\rangle_{0}$ a semi-invariant with respect to the probability measure $P_{0}$. The definition and properties of semi-invariants are described in literature, see for example Malyshev (1980) and references in it.

\section{Notation 5.1}

For a random variable $Y$ on $(\Omega, \Sigma)$ and a sequence $\gamma=\left(B_{1}, \ldots, B_{n}\right)$ of elements of $\mathfrak{B}$ denote:

$$
\left\langle Y, \Phi_{\gamma}\right\rangle_{0}=\left\langle Y, \Phi_{B_{1}}, \Phi_{B_{2}}, \ldots, \Phi_{B_{n}}\right\rangle_{0}
$$

If $n=0$, then $\left\langle Y, \Phi_{\gamma}\right\rangle_{0}=\langle Y\rangle_{0}$.

\section{Definition 5.2}

A sequence $\gamma=\left(B_{0}, B_{1}, \ldots, B_{n}\right)$ of subsets of $\mathbb{Z}^{v}$ is called connected if the following graph is connected:

a) its set of vertices is $\{0,1, \ldots, n\}$;

b) it has no loops; a pair of distinct vertices $i, j$ is connected with an edge if and only if $B_{i} \cap B_{j} \neq \varnothing$.

\section{Lemma 5.3}

Suppose $B_{1}, \ldots, B_{n} \in \mathfrak{B}$ and the sequence $\left(Q, B_{1}, \ldots, B_{n}\right)$ is not connected. Then

$$
\left\langle I_{A}, \Phi_{B_{1}}, \ldots, \Phi_{B_{n}}\right\rangle_{0}=0
$$

\section{Proof}

Proof can be found in Malyshev and Minlos (1991), page 29 (property C).

\subsection{Families}

\section{Definition 5.4}

1) A family (of elements of $\mathfrak{B}$ ) is a set of pairs $\Gamma=$ $\left\{\left(C_{1}, n_{1}\right), \ldots,\left(C_{k}, n_{k}\right)\right\}$, where $C_{1}, \ldots, C_{k}$ are distinct elements of $\mathfrak{B}$ and $n_{i} \geq 1$ for each $i=1, \ldots, k$.

2) The number $n_{i}$ is called the multiplicity of element $C_{i}$ in the family $\Gamma(i=1, \ldots, k)$.
3) We denote the length of the family $\Gamma$ as

$$
\begin{aligned}
& |\Gamma|=n_{1}+n_{2}+\ldots+n_{k} \text { and } \Gamma !=n_{1} ! \cdot n_{2} ! \cdot \ldots \cdot n_{k} ! \\
& \text { 4) } u_{i}(\Gamma)=\sum_{j: C_{i} \cap C_{j} \neq \varnothing} n_{j} \text {. Clearly, } u_{i}(\Gamma) \geq n_{i} .
\end{aligned}
$$

5) For a random variable $Y$ on $(\Omega, \Sigma)$ denote

$$
\left\langle Y, \Phi_{\Gamma}\right\rangle_{0}=\langle Y, \underbrace{\Phi_{C_{1}}, \ldots, \Phi_{C_{1}}}_{n_{1} \text { times }}, \ldots, \underbrace{\Phi_{C_{k}}, \ldots, \Phi_{C_{k}}}_{n_{k} \text { times }}\rangle_{0} .
$$

6) The family $\Gamma=\left\{\left(C_{1}, n_{1}\right), \ldots,\left(C_{k}, n_{k}\right)\right\}$ is called $\boldsymbol{Q}$ connected if the sequence $\left(Q, C_{1}, \ldots, C_{k}\right)$ is connected.

\section{Definition 5.5}

We say that a sequence $\gamma=\left(B_{1}, \ldots, B_{n}\right)$ reduces to a family $\left\{\left(C_{1}, n_{1}\right), \ldots,\left(C_{k}, n_{k}\right)\right\}$, if $C_{1}, \ldots, C_{k}$ are the elements $B_{1}, \ldots, B_{n}$ written without repetitions and each $n_{i}$ is the number of times that $C_{i}$ is repeated in $\gamma$.

Clearly, $n_{1}+\ldots+n_{k}=n$.

\section{Lemma 5.6}

For each family $\Gamma$ of length $n$ there are $\frac{n !}{\Gamma !}$ sequences that reduce to $\Gamma$.

\section{Proof}

The lemma follows from the definitions and combinatorics.

\section{Lemma 5.7}

If a sequence $\gamma$ reduces to a family $\Gamma$, then

$$
\left\langle Y, \Phi_{\gamma}\right\rangle_{0}=\left\langle Y, \Phi_{\Gamma}\right\rangle_{0} .
$$

\section{Proof}

The lemma follows from the fact that the value of a semi-invariant does not depend on the order of random variables.

The following two subsections contain some estimates, which will be used to prove convergence in Section 6. We are not looking for most accurate estimates, since we are not interested in the speed of the convergence.

\subsection{Estimating the Number of Families}

\section{Lemma 5.8}

Fix a natural number $n>1$. The number of all $Q$ connected families $\Gamma$ of length $n$ is less than $2^{2 q}\left(2(8 v)^{2 r}\right)^{n}$. 


\section{Proof}

Denote * the operation of concatenation of two arbitrary sequences. Thus, for sequences $\alpha=\left(\alpha_{0}, \ldots, \alpha_{m}\right)$ and $\tau=\left(\tau_{0}, \ldots, \tau_{l}\right)$ :

$$
\alpha * \tau=\left(\alpha_{0}, \ldots, \alpha_{m}, \tau_{0}, \ldots, \tau_{l}\right)
$$

We also denote:

$$
\alpha * \mid{ }_{j} \tau=\left(\alpha_{0}, \ldots, \alpha_{j}, \tau_{0}, \ldots, \tau_{l}, \alpha_{j+1}, \ldots, \alpha_{m}\right) ; j=1, \ldots, m,
$$

which means inserting the sequence $\tau$ between two consecutive elements of $\alpha$. Clearly:

$$
\left.\alpha *\right|_{m} \tau=\alpha * \tau
$$

Denote $h=2 q$ and $p=2 r$. Fix the associated track $t r_{Q}$ of the set $Q$ :

$$
t r_{Q}=\left(\boldsymbol{t}_{00}, \ldots, \boldsymbol{t}_{0 h}\right)
$$

For each $Q$-connected family $\Gamma$ we will construct a sequence $\operatorname{Tr}_{\Gamma}$ as follows. Consider a $Q$-connected family $\Gamma=\left\{\left(C_{1}, n_{1}\right), \ldots,\left(C_{k}, n_{k}\right)\right\}$ of length $n$. By induction on $j$ $(j=0,1, \ldots, k)$ we will construct a track $T r_{j}$ and a sequence of pairs $\widetilde{T r}_{j}$.

$$
\text { Case } j=0 \text {. }
$$

Define:

$$
\operatorname{Tr}_{0}=t r_{Q} \text { and } \widetilde{\operatorname{Tr}_{0}}=\left(\left(a_{00}, \boldsymbol{t}_{00}\right), \ldots,\left(a_{0 h}, \boldsymbol{t}_{0 h}\right)\right)
$$

where $a_{0 i}=\left\{\begin{array}{l}0 \text { if } \boldsymbol{t}_{0 i} \notin Q, \\ 1 \text { if } \boldsymbol{t}_{0 i} \in Q .\end{array}\right.$

\section{Inductive Step}

Assume we have constructed a track $T_{j-1}$ and a sequence $\widetilde{T r}_{j-1}$. Due to connectedness,

$$
\left(Q \cup C_{1} \cup \ldots \cup C_{j-1}\right) \cap\left(\bigcup_{i=j}^{k} C_{i}\right) \neq \varnothing
$$

Select $\boldsymbol{t}_{\beta_{j}}$ as the first element of the track $T r_{j-1}$ that belongs to this intersection (but not the start element of $\left.T r_{j-1}\right)$. Without loss of generality we can assume:

$$
\boldsymbol{t}_{\beta_{j}} \in\left(Q \cup C_{1} \cup \ldots \cup C_{j-1}\right) \cap C_{j} .
$$

Choose an extended track $t r_{j}$ of the set $C_{j}$ : $t r_{j}=\left(\boldsymbol{t}_{j 0}, \boldsymbol{t}_{j 1}, \ldots, \boldsymbol{t}_{j \mathrm{p}}\right)$. Since the track is a closed path, we can assume it starts at the point $\boldsymbol{t}_{\beta_{j}}: \boldsymbol{t}_{j 0}=\boldsymbol{t}_{\beta_{j}}$. Define

$$
\operatorname{Tr}_{j}=\left.T r_{j-1} *\right|_{\beta j}\left(\boldsymbol{t}_{j 1}, \ldots, \boldsymbol{t}_{j p}\right) .
$$

Denote $T r_{j-1}^{\prime}$ the sequence $\widetilde{T r}_{j-1}$, where the element with index $\beta_{j}$ is changed from $(a, t)$ to $\left(n_{j}+1, t\right)$.

Define

$$
\widetilde{T r}_{j}=\left.\operatorname{Tr}_{j-1}^{\prime} *\right|_{\beta j}\left(\left(a_{j 1}, \boldsymbol{t}_{j 1}\right), \ldots,\left(a_{j p}, \boldsymbol{t}_{j p}\right)\right)
$$

where $a_{j i}=\left\{\begin{array}{l}0 \text { if } \boldsymbol{t}_{j i} \notin C_{j}, \\ 1 \text { if } \boldsymbol{t}_{j i} \in C_{j} .\end{array}\right.$

At the end of this process we take $T r_{\Gamma}=\widetilde{T r}_{k}$.

Next we estimate the number of sequences of the form $T r_{\Gamma}$. A sequence $T r_{\Gamma}=\widetilde{T r}_{k}$ has $(1+h+k p)$ elements; each of these elements is a pair of a number and a point in $\mathbb{Z}^{v}$. The $(1+h)$ points of $t r_{Q}$ are fixed. For each of the remaining $k p$ points there are at most $2 v$ choices, so there are at most $(2 v)^{k p} \leq(2 v)^{n p}$ ways to choose second elements of the pairs in $\operatorname{Tr}_{\Gamma}$.

Next we estimate the number of choices for first elements of the pairs in $\operatorname{Tr}_{\Gamma}$. There are $\left(\begin{array}{c}n+k-1 \\ k-1\end{array}\right)$ ways to split $n$ into a sum $n=n_{1}+\ldots+n_{k}$. If $n_{1}, \ldots, n_{k}$ are chosen, there are $\left(\begin{array}{c}h+k p \\ k\end{array}\right)$ ways to assign the numbers $\left(n_{1}+1\right), \ldots,\left(n_{k}+1\right)$ to first elements of some of the pairs in $\operatorname{Tr}_{\Gamma}$. There are $2^{k p-k}$ ways to assign 0 and 1 to first elements of the remaining pairs (since the points of $t r_{Q}$ are fixed). So the total number of choices for first elements of the pairs in $\operatorname{Tr}_{\Gamma}$ is at most:

$$
\begin{aligned}
& \sum_{k=1}^{n}\left(\begin{array}{c}
n+k-1 \\
k-1
\end{array}\right) \cdot\left(\begin{array}{c}
h+k p \\
k
\end{array}\right) \cdot 2^{k p-k} \\
& \leq \sum_{k=1}^{n} 2^{n+k-1} \cdot 2^{h+k p} \cdot 2^{k p-k}=2^{n+h-1} \sum_{k=1}^{n} 2^{2 k p} \\
& \leq 2^{n+h-1} \sum_{j=1}^{2 n p} 2^{j}<2^{n+h-1} \cdot 2^{2 n p+1}=2^{h} \cdot 2^{n(2 p+1)} .
\end{aligned}
$$

So the total number of sequences of the form $\operatorname{Tr}_{\Gamma}$ is less than

$$
\begin{aligned}
& (2 v)^{n p} \cdot 2^{h} \cdot 2^{n(2 p+1)}=2^{h}\left(2^{3 p+1} v^{p}\right)^{n} \\
& =2^{h}\left(2(8 v)^{p}\right)^{n}=2^{2 q}\left(2(8 v)^{2 r}\right)^{n} .
\end{aligned}
$$


In order to prove the same estimation for the number of $Q$-connected families $\Gamma$ with $|\Gamma|=n$ it is sufficient to show that a family $\Gamma$ can be restored from a sequence $\operatorname{Tr}_{\Gamma}$ and it is unique (then each $\operatorname{Tr}_{\Gamma}$ corresponds to only one $\Gamma$ ).

Consider one of the previously constructed sequences and denote it $T r$. We will restore the family $\Gamma$ such that $\operatorname{Tr}=\operatorname{Tr}_{\Gamma} . \operatorname{Tr}$ is a sequence of pairs that contains a sequence $\left(\left(b_{00}, \boldsymbol{t}_{00}\right), \ldots,\left(b_{0 h}, \boldsymbol{t}_{0 h}\right)\right)$ corresponding to the sequence $t r_{Q}=\left(\boldsymbol{t}_{00}, \ldots, \boldsymbol{t}_{0 h}\right)$. We can assume that the sequence $\operatorname{Tr}$ starts with the pair $\left(1, \boldsymbol{t}_{00}\right)$.

Denote $k$ the number of the pairs in $T r$, whose first elements are greater than 1 , and denote these first elements $m_{1}, \ldots, m_{k}$. Then $n_{i}=m_{i}-1(i=1, \ldots, k)$, according to our construction. So $\Gamma=\left\{\left(C_{1}, n_{1}\right), \ldots,\left(C_{k}, n_{k}\right)\right\}$. It remains to find the sets $C_{j}(j=1, \ldots, k)$. We will restore each set $C_{j}$ and each sequence $\widetilde{T r}_{j-1}$ by induction on $k-j$.

$$
\text { Case } k-j=0 \text {. }
$$

Then $j=k ; \widetilde{T r}_{k}=\operatorname{Tr}$. Find the last pair in $\widetilde{T r}_{k}$, whose first element is greater than 1 and next $p$ pairs in $\widetilde{T r}_{k}$. Then $\widetilde{T r}_{k-1}$ is obtained from the sequence $\widetilde{T r}_{k}$ by removing all these pairs and $C_{k}$ is the set of all second elements of these pairs that have 1 on the first place.

\section{Inductive Step}

Assume we have restored sets $C_{k}, \ldots, C_{j+1}$ and a sequence $\widetilde{T r}_{j}$. Find the last pair in $\widetilde{T r}_{j}$, whose first element is greater than 1 and next $p$ pairs in $\widetilde{T r}_{j}$. Then $\widetilde{T r}_{j-1}$ is obtained from the sequence $\widetilde{T r}_{j}$ by removing all these pairs and $C_{j}$ is the set of all second elements of these pairs that have 1 on the first place.

Clearly, this induction process produces the unique family $\Gamma=\left\{\left(C_{1}, n_{1}\right), \ldots,\left(C_{k}, n_{k}\right)\right\}$ with $T r_{\Gamma}=T r$.

\subsection{Estimation of Semi-Invariants}

\section{Lemma 5.9}

For any $Q$-connected family $\Gamma=\left\{\left(C_{1}, n_{1}\right), \ldots,\left(C_{k}, n_{k}\right)\right\}$ of length $n$ :

$$
\left|\left\langle I_{A}, \Phi_{\Gamma}\right\rangle_{0}\right| \leq \frac{9}{2} P_{0}(A) \beta^{n}(n+1) 3^{n} \prod_{j=1}^{k}\left(u_{j}+1\right)^{n_{j}},
$$

where each $u_{j}=u_{j}(\Gamma)$.

\section{Proof}

Let us fix a sequence $\gamma=\left(B_{1}, \ldots, B_{n}\right)$ that reduces to $\Gamma$ (such a sequence always exists, according to Lemma 5.6). Denote $\gamma^{\prime}=\left(Q, B_{1}, \ldots, B_{n}\right)$.
For $i=1,2, \ldots, n$ denote $v_{i}$ the number of elements of $\gamma^{\prime}$ that intersect with $B_{i}$. Denote $v_{0}$ the number of elements of $\gamma^{\prime}$ that intersect with $Q$.

$I_{A}$ and all $\Phi_{B_{i}}$ are random variables on $(\Omega, \Sigma)$. According to Lemma 5.7 and Theorem 1 in Malyshev and Minlos (1991), pg. 69,

$$
\left|\left\langle I_{A}, \Phi_{\Gamma}\right\rangle_{0}\right|=\left|\left\langle I_{A}, \Phi_{\gamma}\right\rangle_{0}\right| \leq C_{f} \cdot \frac{3}{2} \prod_{i=0}^{n}\left(3 v_{i}\right) .
$$

Here

$$
C_{f}=\max \left\langle\left\langle I_{A} \cdot \prod_{i \in M_{1}} \Phi_{B_{i}}\right\rangle_{0}\left\langle\prod_{i \in M_{2}} \Phi_{B_{i}}\right\rangle_{0} \ldots\left\langle\prod_{i \in M_{i}} \Phi_{B_{i}}\right\rangle_{0}\right|,
$$

where the maximum is taken over all partitions $\left\{\{0\} \cup M_{1}, M_{2}, \ldots, M_{l}\right\}$ of the set $\{0,1,2, \ldots, n\}$.

Each $\left|\Phi_{B_{i}}\right| \leq \beta$, so $C_{f} \leq P_{0}(A) \beta^{n}$. Hence

$$
\begin{aligned}
& \left|\left\langle I_{A}, \Phi_{\Gamma}\right\rangle_{0}\right| \leq P_{0}(A) \beta^{n} \cdot \frac{3}{2} \prod_{i=0}^{n}\left(3 v_{i}\right)=\frac{3}{2} P_{0}(A) \beta^{n} 3^{n+1} \prod_{i=0}^{n} v_{i} \\
& =\frac{9}{2} P_{0}(A) \beta^{n} 3^{n} \prod_{i=0}^{n} v_{i} .
\end{aligned}
$$

For $j=1,2, \ldots, k$ denote $w_{j}$ the number of elements of $\gamma^{\prime}$ that intersect with $C_{j}$. Each $C_{j}$ is repeated $n_{j}$ times in $\gamma$, so:

$$
\prod_{i=0}^{n} v_{i}=v_{0} \prod_{i=1}^{n} v_{i}=v_{0} \prod_{j=1}^{k}\left(w_{j}\right)^{n_{j}} .
$$

Since $v_{0} \leq n+1$ and each $w_{j} \leq u_{j}+1$, we have:

$$
\begin{gathered}
\prod_{i=0}^{n} v_{i} \leq(n+1) \prod_{j=1}^{k}\left(u_{j}+1\right)^{n_{j}} \text { and } \\
\left|\left\langle I_{A}, \Phi_{\Gamma}\right\rangle_{0}\right| \leq \frac{9}{2} P_{0}(A) \beta^{n} 3^{n}(n+1) \prod_{j=1}^{k}\left(u_{j}+1\right)^{n_{j}} .
\end{gathered}
$$

Lemma 5.10

For any $Q$-connected family $\Gamma=\left\{\left(C_{1}, n_{1}\right), \ldots,\left(C_{k}, n_{k}\right)\right\}$ of length $n$ :

$$
\prod_{j=1}^{k}\left(u_{j}+1\right)^{n_{j}}<(e L)^{n} \prod_{j=1}^{k}\left(n_{j}\right)^{n_{j}},
$$

where each $u_{j}=u_{j}(\Gamma)$.

Proof

$$
u_{j}+1=\left(1+\frac{1}{u_{j}}\right) u_{j}
$$


and

$$
\prod_{j=1}^{k}\left(u_{j}+1\right)^{n_{j}}=\prod_{j=1}^{k}\left(1+\frac{1}{u_{j}}\right)^{n_{j}} \prod_{j=1}^{k} u_{j}^{n_{j}} .
$$

From Definition 5.4 we get $n_{j} \leq u_{j}$, so

$$
\prod_{j=1}^{k}\left(1+\frac{1}{u_{j}}\right)^{n_{j}} \leq \prod_{j=1}^{k}\left(1+\frac{1}{n_{j}}\right)^{n_{j}}<\prod_{j=1}^{k} e=e^{k} \leq e^{n}
$$

Thus,

$$
\prod_{j=1}^{k}\left(1+\frac{1}{u_{j}}\right)^{n_{j}}<e^{n} .
$$

By Theorem 4.1 in Kachapova and Kachapov (2016) we have:

$$
\sum_{j=i}^{k}\left(n_{j} \ln \frac{u_{j}}{n_{j}}\right) \leq n \cdot \ln L
$$

Since

$$
\prod_{j=1}^{k}\left(\frac{u_{j}}{n_{j}}\right)^{n_{j}}=e^{\sum_{j=1}^{k}\left(n_{j} \ln \frac{u_{j}}{n_{j}}\right)},
$$

we imply from (7):

$$
\prod_{j=1}^{k}\left(\frac{u_{j}}{n_{j}}\right)^{n_{j}} \leq e^{n \ln L}=L^{n} .
$$

So

$$
\prod_{j=1}^{k} u_{j}^{n_{j}} \leq L^{n} \prod_{j=1}^{k} n_{j}^{n_{j}}
$$

From (5), (6) and (8) we get:

$$
\prod_{j=1}^{k}\left(u_{j}+1\right)^{n_{j}}<e^{n} L^{n} \prod_{j=1}^{k} n_{j}^{n_{j}}=(e L)^{n} \prod_{j=1}^{k} n_{j}^{n_{j}} \cdot \text { 口 }
$$

\section{Lemma 5.11}

Suppose $\Gamma$ is a $Q$-connected family of length $n$ and $n>3$. Then

$$
\left|\left\langle I_{A}, \Phi_{\Gamma}\right\rangle_{0}\right|<P_{0}(A) \beta^{n}\left(3 e^{2} L\right)^{n}(n+1) \Gamma !
$$

\section{Proof}

By Lemmas 5.9 and 5.10,

$$
\begin{aligned}
& \left|\left\langle I_{A}, \Phi_{\Gamma}\right\rangle_{0}\right| \leq \frac{9}{2} P_{0}(A) \beta^{n}(n+1) 3^{n} \prod_{j=1}^{k}\left(u_{j}+1\right)^{n_{j}} \\
& <\frac{9}{2} P_{0}(A) \beta^{n}(n+1) 3^{n}(e L)^{n} \prod_{j=1}^{k} n_{j}^{n_{j}} .
\end{aligned}
$$

By Stirling formula, $q^{q}<\frac{1}{\sqrt{2 \pi q}} q ! e^{q}$ for any natural number $q>0$. So

$$
\begin{aligned}
& \left|\left\langle I_{A}, \Phi_{\Gamma}\right\rangle_{0}\right|<\frac{9}{2} P_{0}(A) \beta^{n}(n+1)(3 e L)^{n} \prod_{j=1}^{k}\left(\frac{1}{\sqrt{2 \pi n_{j}}} n_{j} ! e^{n_{j}}\right) \\
& \leq \frac{9}{2} P_{0}(A) \beta^{n}(3 e L)^{n}(n+1) \frac{1}{(2 \pi)^{\frac{k}{2}} \sqrt{n_{1} n_{2} \ldots n_{k}}} e^{n} \prod_{j=1}^{k} n_{j} ! \\
& =P_{0}(A) \beta^{n}\left(3 e^{2} L\right)^{n}(n+1) \frac{9}{2(2 \pi)^{\frac{k}{2}} \sqrt{n_{1} n_{2} \ldots n_{k}}} \Gamma !
\end{aligned}
$$

since $\Gamma !=\prod_{j=1}^{k} n_{j}$ !. It remains to prove:

$$
\frac{9}{2(2 \pi)^{\frac{k}{2}} \sqrt{n_{1} n_{2} \ldots n_{k}}}<1
$$

Consider two cases.

Case 1. $k=1$.

Then $n_{1}=n \geq 4,(2 \pi)^{k / 2}=\sqrt{2 \pi}>2.5$ and

$$
\frac{9}{2(2 \pi)^{\frac{k}{2}} \sqrt{n_{1} n_{2} \ldots n_{k}}}=\frac{9}{2 \sqrt{2 \pi} \sqrt{n_{1}}}<\frac{9}{2 \times 2.5 \times 2}<1 .
$$

Case 2. $k \geq 2$.

Then $(2 \pi)^{k / 2} \geq 2 \pi>6$ and $\frac{9}{2(2 \pi)^{k / 2} \sqrt{n_{1} n_{2} \ldots n_{k}}}$ $<\frac{9}{2 \cdot 6 \cdot 1}<1$.

\section{Proof of the Existence Theorem}

Here we use results from the previous section to prove Theorem 2.6.

\section{Notation 6.1}

Denote

$$
J_{A}(N, n)=\sum_{\Gamma} \frac{1}{\Gamma !}\left\langle I_{A}, \Phi_{\Gamma}\right\rangle_{0},
$$

where the sum is taken over all $Q$-connected families $\Gamma=$ $\left\{\left(C_{1}, n_{1}\right), \ldots,\left(C_{k}, n_{k}\right)\right\}$ of length $n$ such that each $C_{i} \subseteq \Lambda_{N}$. 


\section{Lemma 6.2}

Suppose $0 \leq \beta \leq \beta_{0}$. Then

$$
P_{N}(A)=\sum_{n=0}^{\infty} J_{A}(N, n)
$$

\section{Proof}

Fix $\beta$ such that $0 \leq \beta \leq \beta_{0}$. We use the method from Malyshev and Minlos (1991), page 34. For real $x, z$ define

$$
f(x, z)=\ln \left\langle\exp \left(x I_{A}+z U_{N}\right)\right\rangle_{0} .
$$

By the definition of semi-invariants:

$$
\begin{aligned}
& \frac{\left\langle I_{A} e^{z U_{N}}\right\rangle_{0}}{\left\langle e^{z U_{N}}\right\rangle_{0}}=\left.\frac{\partial}{\partial x} f(x, z)\right|_{x=0}=\sum_{n=0}^{\infty} \frac{z^{n}}{n !}\langle I_{A}, \underbrace{U_{N}, \ldots, U_{N}}_{n \text { times }}\rangle_{0} \\
& =\sum_{n=0}^{\infty} \frac{z^{n}}{n !}\left\langle I_{A}, \sum_{B_{1} \in \mathfrak{B}, B_{1} \subseteq \Lambda_{N}} \Phi_{B_{1}}, \ldots, \sum_{B_{n} \in \mathfrak{B}, B_{n} \subseteq \Lambda_{N}} \Phi_{B_{n}}\right\rangle_{0} \\
& =\sum_{n=0}^{\infty} \frac{z^{n}}{n !} \sum_{\substack{B_{1}, \ldots, B_{n}, \\
\text { each } B_{i} \in \mathfrak{B}, B_{i} \subseteq \Lambda_{N}}}\left\langle I_{A}, \Phi_{B_{1}}, \ldots, \Phi_{B_{n}}\right\rangle_{0} .
\end{aligned}
$$

Thus,

$$
\frac{\left\langle I_{A} e^{z U_{N}}\right\rangle_{0}}{\left\langle e^{z U_{N}}\right\rangle_{0}}=\sum_{n=0}^{\infty} \frac{z^{n}}{n !} \sum_{\gamma}\left\langle I_{A}, \Phi_{\gamma}\right\rangle_{0},
$$

where the inner sum is taken over all sequences $\gamma=\left\{B_{1}, \ldots, B_{n}\right\}$ such that each $B_{i} \in \mathfrak{B}$ and $B_{i} \subseteq \Lambda_{N}$.

Substituting $z=1$ and using the definition of $P_{N}$, we get:

$$
P_{N}(A)=\frac{\left\langle I_{A} e^{U_{N}}\right\rangle_{0}}{\left\langle e^{U_{N}}\right\rangle_{0}}=\sum_{n=0}^{\infty} \frac{1}{n !} \sum_{\gamma}\left\langle I_{A}, \Phi_{\gamma}\right\rangle_{0} .
$$

By Lemma 5.3, the inner sum can be taken only over sequences $\gamma=\left(B_{1}, \ldots, B_{n}\right)$ of elements of $\mathfrak{B}$ such that $\left(Q, B_{1}, \ldots, B_{n}\right)$ is connected and each $B_{i} \subseteq \Lambda_{N}$.

By Lemmas 5.6 and 5.7:

$$
\begin{aligned}
& P_{N}(A)=\sum_{n=0}^{\infty} \frac{1}{n !} \sum_{\gamma}\left\langle I_{A}, \Phi_{\gamma}\right\rangle_{0}=\sum_{n=0}^{\infty} \frac{1}{n !} \sum_{\Gamma} \frac{n !}{\Gamma !}\left\langle I_{A}, \Phi_{\Gamma}\right\rangle_{0} \\
& =\sum_{n=0}^{\infty} \sum_{\Gamma} \frac{1}{\Gamma !}\left\langle I_{A}, \Phi_{\Gamma}\right\rangle_{0} .
\end{aligned}
$$

The first inner sum has only sequences $\gamma=\left(B_{1}, \ldots, B_{n}\right)$ such that $\left(Q, B_{1}, \ldots, B_{n}\right)$ are connected. Therefore the last inner sum has only $Q$-connected families $\Gamma$ and

$$
P_{N}(A)=\sum_{n=0}^{\infty} J_{A}(N, n)
$$

\section{Lemma 6.3}

Suppose $0 \leq \beta \leq \beta_{0}$ and $n>3$. Then

$$
\left|J_{A}(N, n)\right| \leq 2^{2 q} P_{0}(A) 0.9^{n}(n+1) .
$$

\section{Proof}

Suppose $0 \leq \beta \leq \beta_{0}$ and $n>3$.

$$
\left|J_{A}(N, n)\right| \leq \sum_{\Gamma} \frac{1}{\Gamma !}\left|\left\langle I_{A}, \Phi_{\Gamma}\right\rangle_{0}\right|,
$$

where the sum is taken over all $Q$-connected families $\Gamma=\left\{\left(C_{1}, n_{1}\right), \ldots,\left(C_{k}, n_{k}\right)\right\}$ of length $n$ (we omit the restriction $C_{i} \subseteq \Lambda_{N}$ ). By Lemma 5.8 the number of addends in this sum is less than $2^{2 q}\left(2(8 v)^{2 r}\right)^{n}$.

By Lemma 5.11,

$$
\left|\left\langle I_{A}, \Phi_{\Gamma}\right\rangle_{0}\right| \leq P_{0}(A)(n+1) \beta^{n}\left(3 e^{2} L\right)^{n} \Gamma !
$$

and

$$
\begin{aligned}
& \left|J_{A}(N, n)\right| \leq \sum_{\Gamma} \frac{1}{\Gamma !} P_{0}(A)(n+1) \beta^{n}\left(3 e^{2} L\right)^{n} \Gamma ! \\
& =\sum_{\Gamma} P_{0}(A)(n+1) \beta^{n}\left(3 e^{2} L\right)^{n} \\
& \leq 2^{2 q}\left(2(8 v)^{2 r}\right)^{n} P_{0}(A)(n+1) \beta^{n}\left(3 e^{2} L\right)^{n} \\
& =2^{2 q} P_{0}(A)(n+1)\left[\beta \cdot 6 e^{2} L(8 v)^{2 r}\right]^{n} .
\end{aligned}
$$
have:

Since $0 \leq \beta \leq \beta_{0}$, then by the Definition 2.3 of $\beta_{0}$ we

$$
\begin{gathered}
\beta \cdot 6 e^{2} L(8 v)^{2 r} \leq \beta_{0} \cdot 6 e^{2} L(8 v)^{2 r}=\frac{6 e^{2}}{50}<0.9 \text { and } \\
\left|J_{A}(N, n)\right| \leq 2^{2 q} P_{0}(A)(n+1) 0.9^{n} .
\end{gathered}
$$

\section{Lemma 6.4}

Suppose $0 \leq \beta \leq \beta_{0}$. Then the series

$$
\sum_{n=0}^{\infty} J_{A}(N, n)
$$


converges absolutely and uniformly for all $N \in \mathbb{N}_{Q}$.

\section{Proof}

This follows from Lemma 6.3, since the series $\sum_{n=0}^{\infty} 0.9^{n}(n+1)$ converges. ㅁ

\section{Lemma 6.5}

Suppose $0 \leq \beta \leq \beta_{0}$. Then for any $n$ the following limit exists:

$$
\lim _{N \rightarrow \infty} J_{A}(N, n) .
$$

\section{Proof}

Denote $\mathbf{0}=(0, \ldots, 0)$, the origin in $\mathbb{Z}^{v}$ and $d=\min \{\|\boldsymbol{t}-\mathbf{0}\| \mid \boldsymbol{t} \in Q\} ; d$ is the distance of the set $Q$ from the origin.

For any $n$ denote $M_{n}=r(n+1)+q+d$. We will prove that for any $N \geq M_{n}$ :

$$
J_{A}(N, n)=J_{A}\left(M_{n}, n\right)
$$

Then for any fixed $n, \lim _{N \rightarrow \infty} J_{A}(N, n)$ exists and $\lim _{N \rightarrow \infty} J_{A}(N, n)=J_{A}\left(M_{n}, n\right)$.

\section{Proof of (9)}

Consider any $N \geq M_{n}$. Then $\Lambda_{M_{n}} \subseteq \Lambda_{N}$, which implies the following.

If $\Gamma=\left\{\left(C_{1}, n_{1}\right), \ldots,\left(C_{k}, n_{k}\right)\right\}$ is a $Q$-connected family of length $n$ such that each $C_{i} \subseteq \Lambda_{M_{n}}$, then each $C_{i} \subseteq \Lambda_{N}$.

It remains to prove:

If $\Gamma=\left\{\left(C_{1}, n_{1}\right), \ldots,\left(C_{k}, n_{k}\right)\right\}$ is a $Q$-connected family of length $n$ such that each $C_{i} \subseteq \Lambda_{N}$, then each $C_{i} \subseteq \Lambda_{M_{n}}$.

Then from (10) and (11) we imply that $J_{A}(N, n)$ and $J_{A}\left(M_{n}, n\right)$ are sums over the same set of families $\Gamma$. Hence $J_{A}(N, n)=J_{A}\left(M_{n}, n\right)$.

\section{Proof of (11)}

Consider a $Q$-connected family $\Gamma=\left\{\left(C_{1}, n_{1}\right), \ldots,\left(C_{k}, n_{k}\right)\right\}$ of length $n$ such that each $C_{i} \subseteq \Lambda_{N}$. Fix $i=1, \ldots, k$. To prove that $C_{i} \subseteq \Lambda_{M_{n}}$, we fix an arbitrary $t \in C_{i}$ and prove that $\boldsymbol{t} \in \Lambda_{M_{n}}$.

For some $\boldsymbol{t}_{0} \in Q, d=\left\|\boldsymbol{t}_{0}-\mathbf{0}\right\|$. Since $\left(Q, C_{1}, \ldots, C_{n}\right)$ is connected, there are a subsequence $\left(C_{j_{1}}, \ldots, C_{j_{m}}\right)$ of the sequence $\left(C_{1}, \ldots, C_{k}\right)$ and points $\boldsymbol{t}_{1}, \boldsymbol{t}_{2}, \ldots, \boldsymbol{t}_{m}, \boldsymbol{t}_{m+1}$ such that:

$$
\begin{aligned}
& \boldsymbol{t}_{1} \in C_{i} \cap C_{j_{1}}, \boldsymbol{t}_{2} \in C_{j 1} \cap C_{j_{2}}, \ldots, \boldsymbol{t}_{m} \in C_{j_{m-1}} \cap C_{j_{m}}, \text { and } \\
& \boldsymbol{t}_{m+1} \in C_{j_{m}} \cap Q .
\end{aligned}
$$

Then $m \leq k \leq n$ and

$$
\begin{aligned}
& \|\boldsymbol{t}-\mathbf{0}\| \leq\left\|\boldsymbol{t}-\boldsymbol{t}_{1}\right\|+\left\|\boldsymbol{t}_{1}-\boldsymbol{t}_{2}\right\|+\ldots+\left\|\boldsymbol{t}_{m}-\boldsymbol{t}_{m+1}\right\|+\left\|\boldsymbol{t}_{m+1}-\boldsymbol{t}_{0}\right\| \\
& +\left\|\boldsymbol{t}_{0}-\mathbf{0}\right\| \leq r(m+1)+q+d \leq r(n+1)+q+d=M_{n} . \\
& \text { So }\|\boldsymbol{t}-\mathbf{0}\| \leq M_{n} \text { and } \boldsymbol{t} \in \Lambda_{M_{n}} . \quad \square
\end{aligned}
$$

\section{Proof of Theorem 2.6}

\section{Proof}

1) Suppose $0 \leq \beta \leq \beta_{0}$. By Lemma 6.2,

$$
P_{N}(A)=\sum_{n=0}^{\infty} J_{A}(N, n)
$$

Due to Lemmas 6.4, 6.5 and a property of uniform convergence, $\lim _{N \rightarrow \infty} P_{N}(A)$ exists and

$$
P_{\beta, Q}(A)=\lim _{N \rightarrow \infty} P_{N}(A)=\sum_{n=0}^{\infty} \lim _{N \rightarrow \infty} J_{A}(N, n)
$$

2) To prove that $P_{\beta}$ is a probability measure on $\left(\Omega, \Sigma_{Q}\right)$ we check three probability axioms.

$$
\begin{aligned}
& P_{\beta, Q}(\varnothing)=\lim _{N \rightarrow \infty} P_{N}(\varnothing)=\lim _{N \rightarrow \infty} 0=0 . \\
& P_{\beta, Q}(\Omega)=\lim _{N \rightarrow \infty} P_{N}(\Omega)=\lim _{N \rightarrow \infty} 1=1 .
\end{aligned}
$$

To complete the proof, it remains to check the axiom of $\sigma$-additivity. Consider a sequence of disjoint events $A_{i} \in \Sigma_{Q}(i=1,2, \ldots)$ and denote $D=\bigcup_{i=1}^{\infty} A_{i}$. By (12):

$$
P_{N}(D)=\sum_{i=1}^{\infty} P_{N}\left(A_{i}\right)=\sum_{i=1}^{\infty} \sum_{n=0}^{\infty} J_{A_{i}}(N, n)
$$


By Lemma 6.3, for $n>3$ and $i=1,2, \ldots$,

$$
\left|J_{A_{i}}(N, n)\right| \leq 2^{2 q} P_{0}\left(A_{i}\right) 0.9^{n}(n+1)
$$

Since for $0<x<1, \sum_{n=0}^{\infty} x^{n}(n+1)=\frac{1}{(1-x)^{2}}$, we have:

$$
\begin{aligned}
& \sum_{i=1}^{\infty} \sum_{n=0}^{\infty} 2^{2 q} P_{0}\left(A_{i}\right) 0.9^{n}(n+1)=2^{2 q} \sum_{i=1}^{\infty} P_{0}\left(A_{i}\right) \sum_{n=0}^{\infty} 0.9^{n}(n+1) \\
& =2^{2 q} \sum_{i=1}^{\infty} P_{0}\left(A_{i}\right) \frac{1}{(1-0.9)^{2}}=2^{2 q} 100 \sum_{i=1}^{\infty} P_{0}\left(A_{i}\right)=2^{2 q} 100 P_{0}(D) .
\end{aligned}
$$

So the series on the right-hand side of (14) converges absolutely and uniformly for all $N \in \mathbb{N}_{Q}$. Taking a limit in (14) and using (13), we get:

$$
P_{\beta, Q}(D)=\lim _{N \rightarrow \infty} P_{N}(D)=\sum_{i=1}^{\infty} \sum_{n=0}^{\infty} \lim _{N \rightarrow \infty} J_{A_{i}}(N, n)=\sum_{i=1}^{\infty} P_{\beta, Q}\left(A_{i}\right) .
$$

This proves that:

$$
P_{\beta, Q}\left(\bigcup_{i=1}^{\infty} A_{i}\right)=\sum_{i=1}^{\infty} P_{\beta, Q}\left(A_{i}\right) .
$$

\section{Conclusion}

In this paper we show that in the case of high temperatures it is possible to provide a straightforward and rigorous proof of existence of Gibbs measure. This is important for foundations of statistical mechanics because many of its models are based on the infinite Gibbs measure. We generalised these models to a single interaction model. For a special case of the interaction model we included a version of the central limit theorem; it describes the distribution of a renormgroup transformed field when the volume infinitely increases; this distribution is proved to be an independent Gaussian distribution.

Thus, our paper contributes to foundations of statistical mechanics. Rigorous construction of mathematical foundations of statistical mechanics is necessary to ensure consistency of this theory and validity of its applications. For example, recent applications of statistical mechanics in nanotechnology require especially rigorous and accurate mathematical approach. Further discussion of the role of fundamental mathematics can be found, for example, in (Kachapova, 2014).

Future directions of research on the topic of this paper include possible applications of the results in the theory of soft matter, more general cases of the interaction model and their properties, and more general versions of the Existence theorem (Theorem 2.6) and Convergence theorem (Theorem 3.3).

\section{Acknowledgement}

The authors thank the Editor in Chief and anonymous Reviewer for their valuable comments that helped to improve this paper.

\section{Author's Contributions}

Farida Kachapova and Ilias Kachapov: contributed to the preparation, development and publication of the manuscript.

\section{Ethics}

This is a mathematical article; no ethical issues can arise after its publication.

\section{References}

Baxter, R.J., 2008. Exactly Solved Models in Statistical Mechanics. 1st Edn., Dover Publications, ISBN-10: 0486462714. pp: 498.

Dobrushin, R., 1968. Gibbsian random fields for lattice system with pairwise interactions. Funct. Anal. Applic., 77: 292-301. DOI: 10.1007/BF01075681.

Friedli, S. and Y. Velenik, 2017. Statistical Mechanics of Lattice Systems: A Concrete Mathematical Introduction. 1st Edn., Cambridge University Press, ISBN-10: 1107184827. pp: 622.

Georgii, H.O., 2011. Gibbs Measures and Phase Transitions. 1st Edn., Walter De Gruyter, ISBN-13: 9783110250299. pp: 545.

Kachapova, F. and I. Kachapov, 2016. Convergence of renormalization group transformations of Gibbs random field. J. Math. Stat., 12: 135-151. DOI: $10.3844 /$ jmssp.2016.135.151.

Kachapova, F. and I. Kachapov, 2017. Interaction model in statistical mechanics. J. Math. Stat., 13: 339-346. DOI: $10.3844 /$ jmssp.2017.339.346.

Kachapova, F., 2014. On the importance of pure mathematics. J. Math. Stat., 10: 421-422. DOI: $10.3844 /$ jmssp.2014.421.422.

Kadanoff, L., 1966. Scaling laws for using models near Tc. Phys., 2: 263-272. DOI: 10.1103/PhysicsPhysiqueFizika.2.263.

Kashapov, I., 1977. Structure of ground states in threedimensional Ising model with three-step interaction. Theor. Math. Phys., 33: 110-118. DOI: $10.1007 /$ BF01039015.

Malyshev, V., 1980. Cluster expansions in lattice models of statistical physics and quantum field theory. Russian Math. Surveys, 35: 1-62. 
Malyshev, V.A. and R.A. Minlos, 1991. Gibbs Random Fields: Cluster Expansions. 1st Edn., Kluwer Academic Publishers. ISBN-10: 079230232X.

Preston, C., 1976. Random Fields. 1st Edn., SpringerVerlag Berlin Heidelberg,

ISBN-13: 978-3540078524. pp: 204.

Ruelle, D., 1999. Statistical Mechanics: Rigorous Results. 1st Edn., World Scientific, ISBN-13: 978-9810238629. pp: 219.
Yang, Y., B. Teng, H. Yang and H. Cui, 2017. Investigation of probability theory on Ising models with different four-spin interactions. Phys. Stat. Mechan. Applic., 483: 243-349.

DOI: 10.1016/J.PHYSA. 2017.04.176.13 